\title{
Evaluating 5G Coverage in 3D Scenarios under Configurable Antenna Beam Patterns
}

\author{
Francisco Jesus ${ }^{1}$, Marco Sousa ${ }^{2,3,4}$ \\ ${ }^{1}$ Instituto Superior de Engenharia de Lisboa \\ ${ }^{2}$ CELFINET, \\ Consultoria em Telecomunicações, Lda. \\ Lisbon, Portugal \\ A42882@alunos.isel.pt \\ marco.sousa@celfinet.com
}

\author{
Filipe Freitas $^{1}$, Pedro Vieira ${ }^{1,3}$ \\ ${ }^{3}$ Instituto de Telecomunicações \\ Lisbon, Portugal \\ ffreitas@cc.isel.ipl.pt \\ pedro.vieira@isel.pt
}

\author{
A. Rodrigues ${ }^{3,4}$ and M. P. Queluz ${ }^{3,4}$ \\ ${ }^{4}$ Instituto Superior Técnico \\ Lisbon, Portugal \\ [ar, paula.queluz]@1x.it.pt
}

\begin{abstract}
Active Antenna Systems (AASs) play a key role in the performance of $5^{\text {th }}$ Generation (5G) networks as they enable the use of Massive Multiple-Input Multiple-Output (mMIMO) and directional beamforming. Besides, AASs can be configured with distinct broadcast beams configurations. In this work, the coverage provided by the broadcast beam configurations of a real AAS is evaluated. A 3-Dimensional (3D) configurable synthetic scenario was proposed to evaluate the resulting 5G coverage from all the possible antenna beam configurations. This analysis revealed that beam configurations with several horizontal beams and one vertical are recommended for urban macro deployments. Moreover, it was demonstrated that the percentage of covered area in a real scenario is approximated by an equivalent synthetic scenario with a Pearson correlation of 0.98. The synthetic scenario has the advantage of not requiring $3 D$ building databases. Finally, an interference analysis in multi-site real scenarios was conducted, where it was verified that some antenna configurations introduce excessive interference for the level of coverage provided.
\end{abstract}

Index Terms-Wireless Networks, 5G, Active Antenna Systems, Antenna Model, Beamforming

\section{INTRODUCTION}

From the conception of the first mobile networks, the telecommunications industry has been seeking technological evolution to maximize the performance of its systems. Nowadays, with the $5^{\text {th }}$ Generation $(5 \mathrm{G})$ systems already in deployment, it is even more critical to incorporate innovative technologies to achieve the full potential of the New Radio (NR) system. That said, an Active Antenna System (AAS) is a key enabling technology to explore the spatial domain's full potential by introducing new degrees of freedom with 3-Dimensional (3D) beamforming [1]. An AAS can produce distinct scenario-based beam configurations by modifying the number of horizontal/vertical beams and the horizontal/vertical Half-Power Beamwidth (HPBW).

Several works have been proposed that evaluate the network impact in terms of coverage, interference, and throughput rates of AASs with multiple beams. In [2], the authors analyzed coverage and throughput rates in 5G networks. However, directional beamforming was approximated by a sectored antenna model. Also, several works consider the hexagonal grid concept and random models based on spatial point process as in [3]. In [4], the authors proposed a beamforming scheme that depends on a random spatial distribution of users.

In this work, the main goal is to evaluate the coverage impact of distinct AAS configurations for broadcast beams, which contain the synchronization signals required for the network access by User Equipments (UEs). The beam configurations were modeled by a recently proposed beamforming antenna model [5]. Then, instead of statistical user distributions, detailed 3D environments were considered, representing the locations and geometry of buildings. Furthermore, both synthetic and real 3D scenarios were evaluated.

The remainder of this paper is organized as follows. Section II presents the fundamental concepts in this work as AASs and $5 \mathrm{G}$ radio propagation. Then, section III introduces a $3 \mathrm{D}$ configurable Manhattan-like scenario that supports an initial analysis of the coverage impact of a real AAS. In section IV, considering real 3D scenarios, a comparison with approximated synthetic scenarios is performed. The real scenarios are then expanded to a macro multi-site deployment, evaluating signal strength coverage and interference. Finally, Section V presents the main conclusions and final remarks.

\section{5G Coverage Estimation}

In this section, the fundamentals for estimating $5 \mathrm{G}$ radio coverage are presented. First, the AAS, used throughout this work, is overviewed, and the beamforming antenna model to estimate the respective radiation pattern is briefly presented. Then, the used $5 \mathrm{G}$ propagation model and the assumptions for the $5 \mathrm{G}$ radio coverage estimation are presented.

\section{A. $5 G$ Active Antenna Systems}

In this work, it was considered a real AAS from Huawei called AAU5613. This Massive Multiple-Input MultipleOutput (mMIMO) macro antenna produces 16 scenario-based beam configurations, which can be characterized on 4 parameters: number of horizontal beams, number of vertical beams, Horizontal Half-Power Beamwidth (H-HPBW), and Vertical Half-Power Beamwidth (V-HPBW). The beam configurations of the Huawei AAU5613 are displayed in Table I [1], which are considered throughout this work. 
TABLE I

BEAm CONFIGURATIONS OF THE ANTENNA HuAWEi AAU5613 [1].

\begin{tabular}{|c|c|c|c|c|}
\hline Pattern & H-HPBW $\left[^{\circ}\right]$ & V-HPBW $\left[{ }^{\circ}\right]$ & $\begin{array}{c}\text { Horizontal } \\
\text { Beams }\end{array}$ & $\begin{array}{c}\text { Vertical } \\
\text { Beams }\end{array}$ \\
\hline 1 & 110 & 6 & 8 & 1 \\
\hline 2 & 90 & 6 & 6 & 1 \\
\hline 3 & 65 & 6 & 6 & 1 \\
\hline 4 & 45 & 6 & 4 & 1 \\
\hline 5 & 25 & 6 & 2 & 1 \\
\hline 6 & 110 & 12 & 8 & 1 \\
\hline 7 & 90 & 12 & 6 & 1 \\
\hline 8 & 65 & 12 & 6 & 1 \\
\hline 9 & 45 & 12 & 4 & 2 \\
\hline 10 & 25 & 12 & 2 & 2 \\
\hline 11 & 15 & 12 & 1 & 2 \\
\hline 12 & 110 & 25 & 8 & 1 \\
\hline 13 & 65 & 25 & 6 & 1 \\
\hline 14 & 45 & 25 & 4 & 1 \\
\hline 15 & 25 & 25 & 2 & 4 \\
\hline 16 & 15 & 25 & 1 & 4 \\
\hline
\end{tabular}

The coverage impact of using different beam configurations can be modeled by considering the antenna radiation pattern. In this work, the antenna real radiation diagrams were unavailable, which was circumvented by using an antenna radiation model. In [5], a beamforming antenna model was proposed, which was used to model the radiation pattern of the Huawei AAU5613 AAS. It is similar to the $3^{\text {rd }}$ Generation Partnership Project (3GPP) antenna model [6] with the advantage of allowing to model multi-beam antennas. Fig. 1 exemplifies the $3 \mathrm{D}$ radiation gain of both patterns 1 and 9 ( $c f$. Table I)

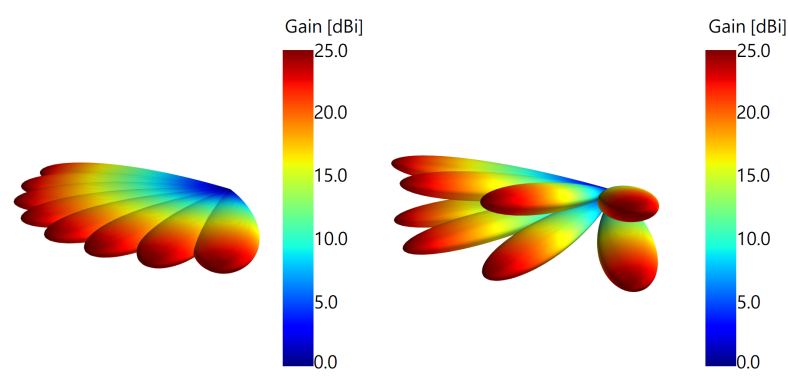

Fig. 1. AAU5613 3D radiation gain of patterns 1 (left) and 9 (right).

\section{B. 5 G Radio Propagation}

After considering the effect of the antenna configurations, the link path loss has to be considered to estimate radio coverage. In this work, the 3 GPP propagation model was used to estimate path loss [6]. Additionally, it was employed a Lineof-Sight $(\mathrm{LoS})$ algorithm to identify the appropriate path loss equation (LoS or Non-Line-of-Sight (NLoS)). In addition, the path link was divided into two components, outdoor and indoor allowing to estimate both outdoor and indoor coverage levels.

In this work, the $5 \mathrm{G}$ radio coverage was evaluated considering the Synchronization Signal Reference Signal Received Power (SS-RSRP). The SS-RSRP is defined as the linear average over the power contributions of the resource elements that carry Secondary Synchronization Signal (SSS) and it is given by [1]:

$$
\text { SS-RSRP }=\mathrm{P}_{\mathrm{TX}, \mathrm{RS}}+\mathrm{G}_{\mathrm{BS}}-\mathrm{A}_{\mathrm{TX}}+\mathrm{G}_{\mathrm{UE}}-\mathrm{PL}
$$

where $P_{T X, R S}$ is the reference signal power in $d B m, G_{B S}$ is the Base Station (BS) antenna gain in $\mathrm{dBi}$ (computed using the beamforming antenna model), $A_{T X}$ is the BS cable losses in $\mathrm{dB}, \mathrm{G}_{\mathrm{UE}}$ is the $\mathrm{UE}$ antenna gain in $\mathrm{dBi}$, and $\mathrm{PL}$ is the path loss in $\mathrm{dB}$.

Concerning the reference signal power, $\mathrm{P}_{\mathrm{TX}, \mathrm{RS}}$, it is calculated as follows (based on [1]):

$$
\mathrm{P}_{\mathrm{TX}, \mathrm{RS}}=\mathrm{P}_{\mathrm{TX}, \max }-10 \log _{10}\left(N_{\mathrm{PRB}}^{\mathrm{BW}, \mu} \times 12\right)
$$

where $\mathrm{P}_{\mathrm{TX}, \max }$ is the maximum transmitted power in $\mathrm{dBm}$, and $N_{\mathrm{PRB}}^{\mathrm{BW}, \mu}$ is the maximum physical resource block allocation in bandwidth BW with numerology $\mu$.

Following, radio coverage percentage was calculated by comparing the SS-RSRP with the receiver sensitivity, $P_{\mathrm{Rx} \text {,Sens }}$, after having margins added. The $P_{\mathrm{Rx} \text {,Sens }}$ is given by [1]:

$$
P_{\mathrm{Rx}, \text { Sens }}=-174+10 \log _{10}(\mathrm{SCS})+N_{\mathrm{F}}+\mathrm{SINR}
$$

where SCS is the Subcarrier Spacing (SCS) in $\mathrm{kHz}$ (according to $\mu), N_{\mathrm{F}}$ is the receiver noise figure, and SINR is the minimum Signal-to-Interference plus Noise Ratio (SINR).

\section{Active Antenna Systems in SynthetiC SCENARIOS}

This section presents a configurable Manhattan-like scenario used to estimate $5 \mathrm{G}$ coverage by considering the beam configurations presented in Table I. Then, the characteristics of beam configurations (e.g., the number of horizontal and vertical beams) are evaluated in terms of achieved coverage in both outdoor and indoor locations.

\section{A. Configurable Manhattan-like scenario}

The Manhattan-like scenario is adjustable in three parameters: average buildings height and width, and average streets width. This initial approach, by using an adjustable synthetic scenario, allows a geometry flexibility of the surrounding environment that a real environment can not provide. Thus, a more comprehensive analysis between the environment geometry and the used beam configuration is possible. The Manhattan-like grid was selected since it simulates the wellknown obstruction experienced in an urban environment.

Afterward, considering any configurable Manhattan-like scenario, a beam configuration using the beamforming model (see section II-A)), the 3GPP path loss model, and the equations from section II-B, the SS-RSRP can be estimated in the whole scenario. An example of the SS-RSRP estimation in a scenario with a buildings' width of $41 \mathrm{~m}$, a buildings' height of $31 \mathrm{~m}$, and a streets' width of $27 \mathrm{~m}$ is depicted in Fig. 2.

Concerning the 3D scenario software development, the Python PyVista module [7] was adopted to generate a structured grid shaped with three arrays containing all the scenario coordinates. The grid, together with an array containing the received power, was applied in the mesh creation method to 


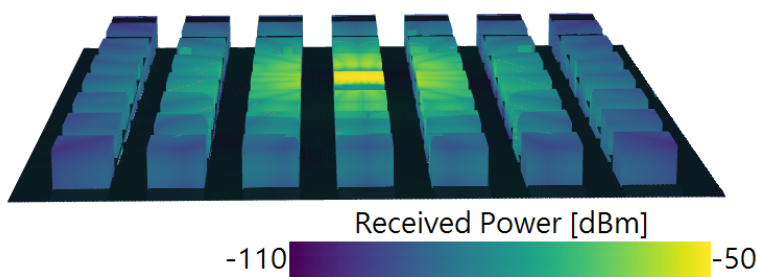

Fig. 2. 3D synthetic scenario heat map with the received power surface and a BS at the center (height of $34 \mathrm{~m}$ ) using radiation pattern 1 .

create a mesh that also considers the received power surface. The simulator code was tested using a Python Unit testing framework called unittest [8] and it is available in a private repository on GitHub.

\section{B. 5G Coverage Analysis Setup}

The $5 \mathrm{G}$ coverage analysis in synthetic scenarios considered a 3-sector macro BS in the top of the buildings operating at a carrier frequency of $3.5 \mathrm{GHz}$. Besides, all the antenna beam configurations (see Table I) were analyzed. Moreover, the configurable Manhattan-like scenario allowed the evaluation of a wide range of environment geometries. In total, 672 unique Manhattan-like geometries were evaluated according to the three scenario parameters (see Table II).

TABLE II

RANGE OF THE MANHATTAN-LIKE CONFIGURABLE PARAMETERS.

\begin{tabular}{|c|c|c|}
\hline Parameter & Range of applied values [m] & Step [m] \\
\hline Building height & 6 to 50 & 4 \\
\hline Building width & 8 to 36 & 4 \\
\hline Street width & 6 to 30 & 4 \\
\hline
\end{tabular}

In the $5 \mathrm{G}$ coverage analysis, the guideline to define the $\mathrm{BS}$ height, $h_{\mathrm{BS}}$, is given by:

$$
h_{\mathrm{BS}}=\left\{\begin{aligned}
18[\mathrm{~m}], & \text { if } h_{\text {build }}<15[\mathrm{~m}] \\
h_{\text {build }}+3[\mathrm{~m}], & \text { if } h_{\text {build }} \geq 15[\mathrm{~m}]
\end{aligned}\right.
$$

where $h_{\text {build }}$ is the average buildings' height. Note that the BS is placed at the center of the scenario.

\section{C. $5 G$ Coverage Analysis Results}

The coverage impact of the antenna beam configurations, considering all the synthetic scenarios, is assessed by grouping the coverage percentage results by the antenna pattern in the box plot illustrated in Fig. 3. Please note that the colors are purely representative, having no underlying meaning.

Regarding Fig. 3, it is worth highlighting the coverage's dependence on the applied radiation pattern. For instance, the median coverage value can be less than $40 \%$ to higher than $70 \%$, depending on the radiation pattern. All the antenna patterns manifest a high dispersion, which indicates a high impact of the scenario characteristics on coverage. However, an expressive quantity of outliers is evident concerning the

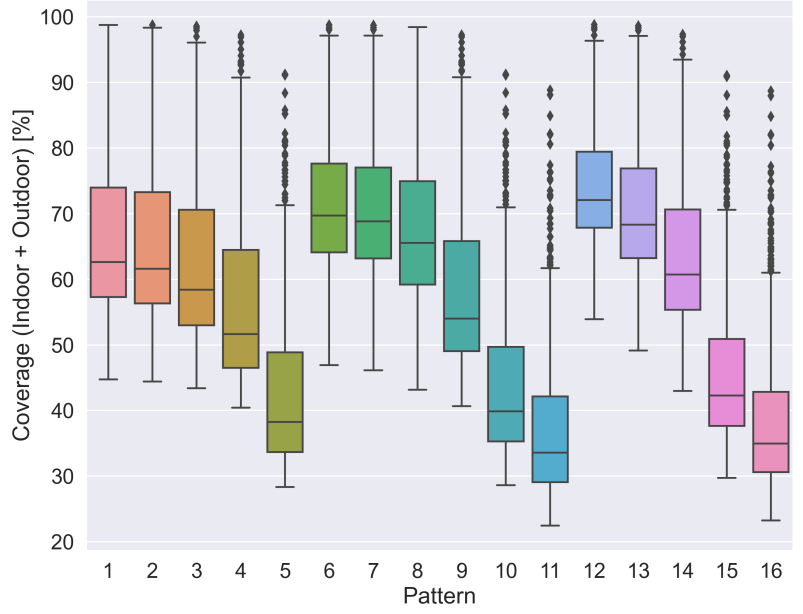

Fig. 3. Coverage percentage (indoor + outdoor) of all scenarios grouped by the antenna pattern.

patterns $5,10,11,15$, and 16 , expressing the sensitivity of those patterns to the environment's geometric characteristics.

On a final note, it is observed that radiation pattern 12 is the only configuration providing a minimum coverage percentage of more than $50 \%$, despite the tested scenario. On the other side, both radiations patterns 11 and 16 obtain a minimum coverage percentage below $25 \%$.

In an attempt to further detail the heterogeneous performance of the radiation patterns, the scenarios coverage results were grouped by each of the antenna parameters, starting with the number of horizontal beams, illustrated in Fig. 4.

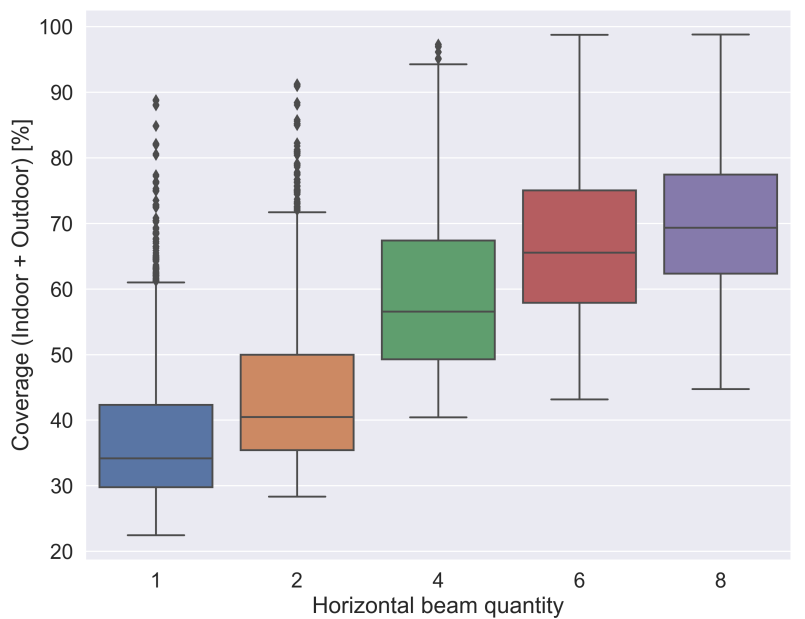

Fig. 4. Coverage percentage (indoor + outdoor) of all scenarios grouped by the number of horizontal beams

As concerns Fig. 4, it is noticeable that radiation patterns with a higher quantity of horizontal beams ensure higher coverage by reaching more horizontal area with adequate signal level (the horizontal component of the considered scenarios is greater than the vertical component). From Fig. 3, it was verified that patterns $5,10,11,15$, and 16 demonstrated a higher sensibility of the obtained coverage compared with 
the remaining patterns. In fact, all these patterns have a low quantity of horizontal beams, which is consistent with the results depicted in Fig. 4; radiation patterns with lower quantity of horizontal beams are the only ones that verify the existence of outlier values for the covered area. The analysis of the percentage of the covered area as a function of $\mathrm{H}$ HPBW was omitted due to similar conclusions regarding Fig. 4, as the total H-HPBW is highly correlate with the number of horizontal beams.

In respect of the V-HPBW, Fig. 5 exhibits the scenarios coverage results grouped by the V-HPBW.

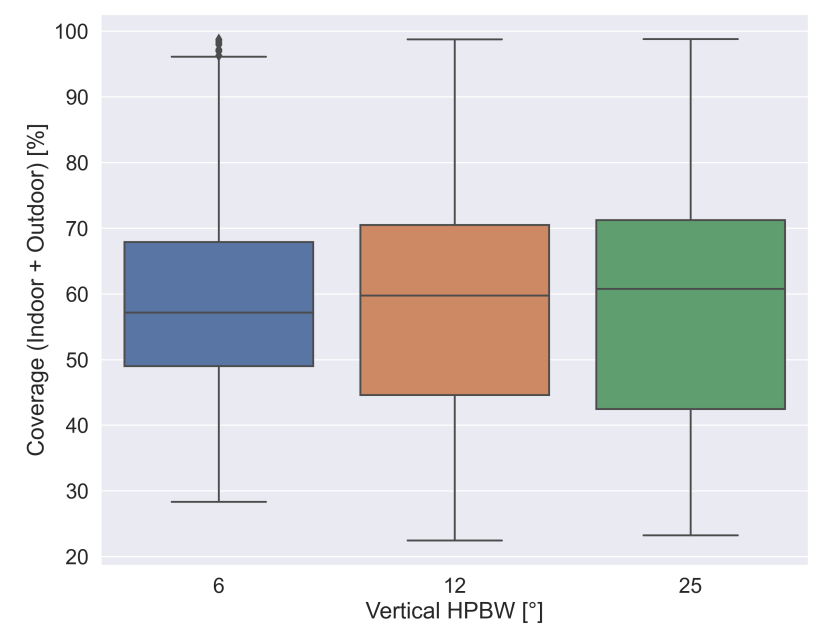

Fig. 5. Coverage percentage (indoor + outdoor) of all scenarios grouped by the vertical HPBW.

Regarding Fig. 5, it is noted that the minimum coverage percentage for the distributions with a V-HPBW of $12^{\circ}$ and $25^{\circ}$ is inferior to the minimum of the distribution with a VHPBW of $6^{\circ}$. However, the first two mentioned distributions include a single-horizontal beam pattern (which are the worstperforming patterns of the 16 available). Despite that, it is noticed that the median and third quartile achieved higher coverage for the wider V-HPBW. Finally, the last parameter, the number of vertical beams, is illustrated in Fig. 6 .

Regarding Fig. 6, one may notice that single-vertical beam radiation patterns ensure more coverage. In contrast, the radiation patterns with four vertical beams intend to provide higher gain on the vertical dimension (not as representative as the horizontal dimension) on specific scenarios focused on a narrower horizontal region.

The previous results evaluated how the covered area was affected by the variation of the main characteristics of the antenna radiation patterns, regardless of the scenarios. The following results attempt to evaluate how the covered area is affected by the variation of the scenarios parameters.

In that sense, to evaluate the scenario parameters' influence on coverage, it was established a factor that characterizes the users' distribution by computing the average height of users for each scenario. Note that this factor involves the three scenario modeling parameters. The average users' height in meters,

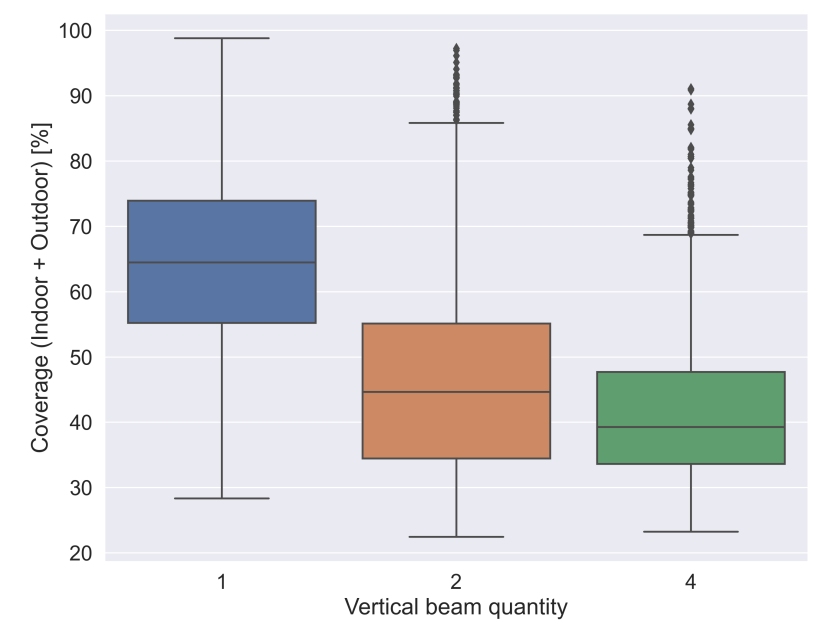

Fig. 6. Coverage percentage (indoor + outdoor) of all scenarios grouped by the number of vertical beams.

$\overline{\mathrm{h}_{\mathrm{UT}}}$, is given by:

$$
\overline{h_{U T}}=\frac{\left(h_{\mathrm{s}} \times \mathrm{s}_{\mathrm{a}} \times \mathrm{u}_{\mathrm{d}}\right)+\sum_{\mathrm{n}=1}^{\mathrm{n}_{\text {floors }}}\left(\mathrm{h}_{\mathrm{n}} \times \mathrm{f}_{\mathrm{a}} \times \mathrm{u}_{\mathrm{d}}\right)}{\mathrm{t}_{\mathrm{a}} \times \mathrm{u}_{\mathrm{d}}}
$$

where $h_{s}$ is the UE height of the users on the street in meters, $\mathrm{u}_{\mathrm{d}}$ is the users density expressed in users per square meter, $\mathrm{s}_{\mathrm{a}}$ is the total street area in square meters, $h_{n}$ is the corresponding floor height in meters, $f_{a}$ is the sum of all buildings' first floor area in square meters, and $t_{a}$ is the total area of the scenario in square meters.

Fig. 7, illustrates the coverage variation for each radiation pattern in function of the $\overline{\mathrm{h}_{\mathrm{UT}}}$.

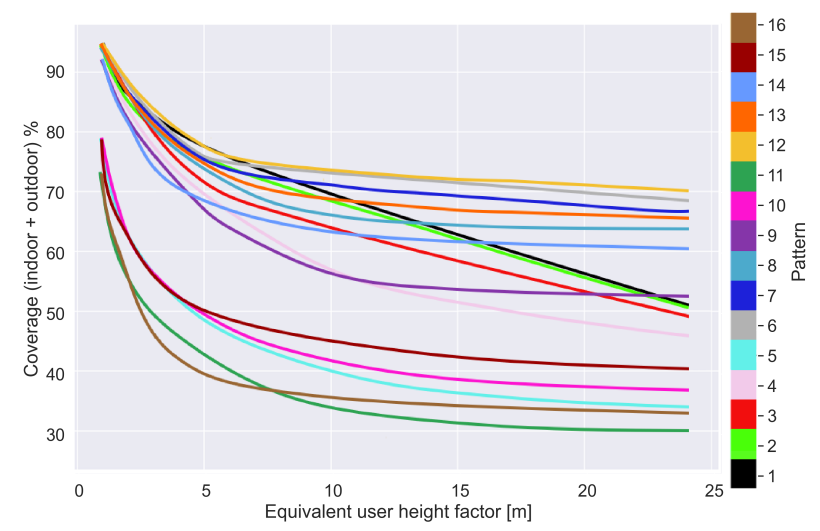

Fig. 7. Coverage variation for each antenna pattern in function of the $\overline{h_{U T}}$ factor.

Concerning Fig. 7, it is noted that when $\overline{\mathrm{h}_{\mathrm{UT}}}$ increases, coverage deteriorates since it represents a more densified (and therefore more complex) environment. Note that in Fig. 7, from 10 meters upwards, the coverage degradation is superior in radiation patterns 1 to 5 , characterized by having a low V-HPBW. Likewise, radiation pattern 11 is also decreasing coverage significantly. However, it stabilizes when the factor reaches 20 meters due to its two vertical beams. 
Overall, radiation pattern 12 generally outperforms the remaining patterns in urban macro scenarios, mainly due to a wide H-HPBW with multiple horizontal beams. Even though having only one vertical beam, a wide V-HPBW maximizes coverage. Additionally, when the average user height increases (scenarios with higher buildings and/or more buildings), the covered area percentage of beam configurations with the narrower V-HPBW monotonically decreases with the user average height increase. On the other side, antenna configurations with a wider V-HPBW, at least for the range of tested values, tend to guarantee a minimum covered area.

\section{Active Antenna Systems in Real Scenarios}

This section introduces real propagation scenarios considering detailed 3D information about building locations and respective geometries. Then, based on the real scenarios, an approximated synthetic scenario is introduced using the configurable Manhattan-like scenario presented in section III. The use of the approximated scenario to emulate a real scenario is evaluated. Finally, the impact of the antenna beam configurations is extended to evaluate interference levels in multi-site real scenarios.

\section{A. 3D Urban Scenarios}

The 5G coverage analysis, in real scenarios, was conducted using a 3D data sample containing two data sources, terrain elevation, and 3D building models, for a restricted urban area in Lisbon [9]. Based on the geospatial data several real scenarios were created taking advantage of the QGIS [10] processing tools and the Geospatial Data Abstraction software Library (GDAL), a translator library for raster and vector geospatial data formats [11].

Fig. 8 presents a real scenario example with the buildings layout, including a building height heat map, corresponding to a real urban environment with an area of $500 \mathrm{~m}$ by $500 \mathrm{~m}$.

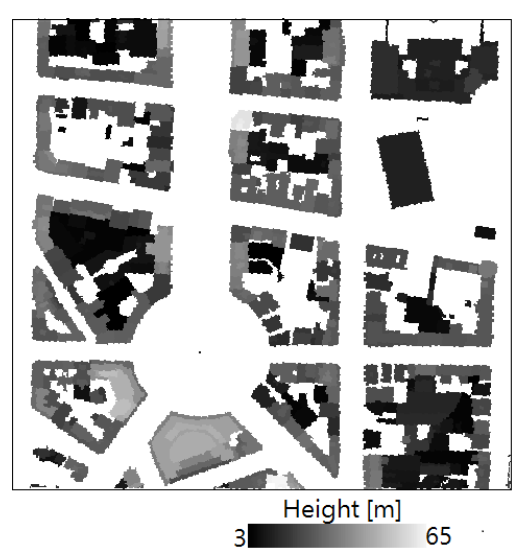

Fig. 8. Example of a real urban scenario with buildings height heat map.

\section{B. Real Scenario vs Approximate Synthetic Scenario}

The main goal in this subsection is to develop an approximate synthetic scenario based on the real scenario building geometry to validate if the conclusions derived from the synthetic scenarios are generalized to real scenarios. The proposed approximate synthetic scenario was compared with the real scenario by considering the percentage of covered area in both, assuming the same location for the BS and considering all possible antenna beam configurations.

Therefore, to obtain the three required parameters (see section III-A) to represent an approximate synthetic model, the average building's height was calculated using both the QGIS processing tools and the 3D building models. Then, by calculating the outdoor area percentage of the real scenario, it was identified the average buildings' width and the streets' width. These three parameters allows to created an approximate synthetic scenario based on a real scenario.

A total of five real scenarios and the respective approximated scenarios were considered, including the real scenario from Fig. 8. The comparison of the percentage of the covered area of both the real and the approximated synthetic scenario demonstrated a good approximation. Overall, a Pearson correlation coefficient of 0.978 , a Spearman correlation coefficient of 0.968, a Mean Absolute Error (MAE) of 3.47, and a Root Mean Square Error (RMSE) of 4.12 were obtained between the percentage of the covered area of the real and the respective approximated synthetic scenario, in all scenarios.

Following, a more detailed analysis to each real scenario was conducted. Considering the real scenario illustrated in Fig. 8, the respective approximate synthetic scenario was characterized by buildings with 21 meters high and 23 meters in width, streets width of 18 meters, and a BS placed at the center with 24 meters high. For all scenarios, all antenna beam configurations were tested, and for simplicity, the three bestperforming patterns are displayed in Table III.

TABLE III

REAL SCENARIO COVERAGE VS APPROXIMATE SYNTHETIC MODEL THREE BEST-PERFORMING RADIATION PATTERNS.

\begin{tabular}{|c|c|c|}
\hline Pattern & Real Scenario Cov. [\%] & Synthetic Scenario Cov. [\%] \\
\hline 12 & 71.68 & 66.17 \\
\hline 13 & 63.85 & 60.60 \\
\hline 6 & 63.08 & 60.02 \\
\hline
\end{tabular}

As it can be observed in Table III, the radiation pattern 12 outperforms the remaining patterns even on real scenarios. This is also verified in the other four tested scenarios.

Overall, it was demonstrated that in the absence of detailed 3D building data, the approximated synthetic scenario allows evaluating the covered area with high accuracy. It has the advantage of only requiring the building average height and width and the street width, which is more easily obtained. Moreover, it validates the conclusions obtained using synthetic scenarios regarding the coverage impact of beam configurations in real scenarios.

\section{Interference Analysis in Real Scenarios}

After carrying out the study on synthetic scenarios, validating the results by comparing five real single-site scenarios to its approximate synthetic model, it was considered besides signal strength analysis, an interference analysis by considering 
scenarios with multiple BSs. The main goal in this subsection is to assess signal strength coverage and SINR. The multi-site scenarios consist of BSs placed on locations and elevations of a real Mobile Network Operator (MNO). Fig. 9 illustrates one of these scenarios.

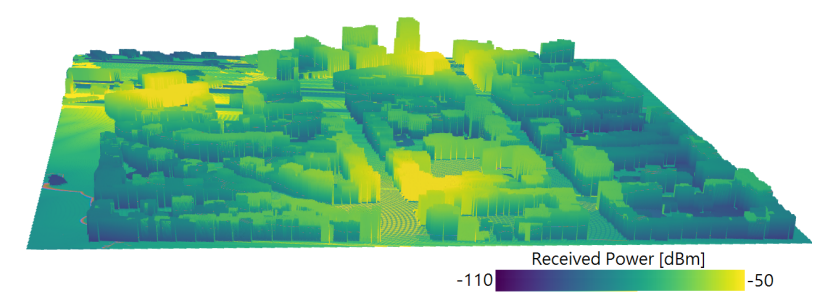

Fig. 9. Real multi-site scenario with 3 BSs using radiation pattern 12 .

In Fig. 9, the 3D building scenario is depicted with a heat map representing the global SS-RSRP obtained by three BSs, all configured with the antenna pattern 12 . This pattern was the best-performing pattern in terms of coverage. However, for the SINR analysis, it did not perform likewise.

Fig. 10 illustrates the Cumulative Density Function (CDF) of the obtained SINR for the scenario in Fig. 9. It represents the top three worst radiation patterns (dashed) and the top three best patterns in terms of SINR.

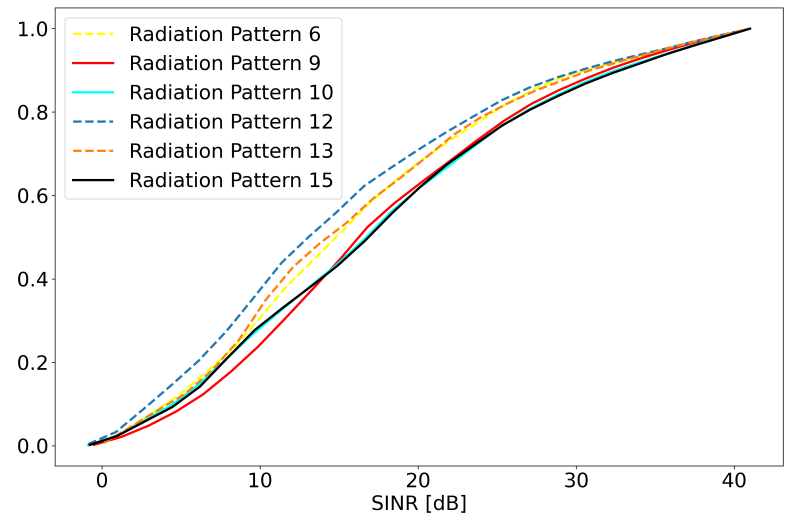

Fig. 10. CDF of the SINR for the real multi-site scenario of Fig. 9.

In Fig. 10, radiation pattern 12 is the worst-performing pattern in terms of SINR, while patterns 13 and 6, which are more directives either in the horizontal or vertical planes, attained higher SINR. Concerning the three best-performing patterns for SINR, these patterns significantly underperform concerning coverage. This result indicates that finding the optimal radiation pattern for a given real multi-site scenario requires contemplating coverage and SINR.

\section{CONCLUSIONS}

In this work, the coverage impact of distinct AAS configurations for broadcast beams has been evaluated. A 3D configurable Manhattan-like scenario was proposed, which implicitly reflects the distribution of UEs for extensive coverage analysis. For urban macro deployments, regarding 5G coverage, there is no incentive on using antenna configurations with multiple vertical beams. Overall, radiation patterns with several horizontal beams and one vertical, with a wide HPBW, are recommended. However, it was demonstrated that the obtained coverage by an antenna configuration could be severely affected by the specific environment geometry. In addition, the conclusions obtained in the synthetic scenarios are expected to be reflected in real scenarios, as it was possible to achieve similar coverage levels between real scenarios and their equivalent synthetic scenario. Including the interference analysis in multi-site scenarios, it was verified that the antenna configurations that maximize coverage also introduced additional interference. Future work is in motion to extend the proposed 3D configurable synthetic scenario for multisite deployments. With this, reliable and extensive data can be produced to develop an antenna configuration algorithm based on Machine Learning (ML), to optimize the antenna configuration pattern for any given scenario geometry.

\section{ACKNOWLEDGMENT}

This work was funded by COMPETE/FEDER, under the project AI4GREEN 16/SI/2019 - I\&DT Empresarial (Projetos Copromoção), through the international project CELTICNEXT/EUREKA (C2018/1-5). Moreover, an acknowledgment is due to CELFINET and Instituto de Telecomunicações (IT) for the support to this work.

\section{REFERENCES}

[1] Sousa, Marco and Alves, André and Vieira, Pedro and Queluz, Maria Paula and Rodrigues, António, "Analysis and Optimization of 5G Coverage Predictions Using a Beamforming Antenna Model and Real Drive Test Measurements," IEEE Access, vol. 9, pp. 101 787-101808, 2021.

[2] Bai, Tianyang and Heath, Robert W., "Coverage and Rate Analysis for Millimeter-Wave Cellular Networks," IEEE Transactions on Wireless Communications, vol. 14, no. 2, pp. 1100-1114, 2015.

[3] Blaszczyszyn, Bartlomiej and Karray, Mohamed, "Spatial Distribution of the SINR in Poisson Cellular Networks With Sector Antennas," IEEE Transactions on Wireless Communications, vol. 15, pp. 1-1, 012015.

[4] Jalal Rachad and Ridha Nasri and Laurent Decreusefond, "A 3D Beamforming Scheme Based on The Spatial Distribution of User Locations," CoRR, vol. abs/2002.04451, 2020. [Online]. Available: https://arxiv.org/abs/2002.04451

[5] Alves, A. and Sousa, M. and Vieira, P. and Queluz, M. P. and Rodrigues, A., "A New 3D Beamforming Antenna Model for 5G Propagation Modeling based on Real Data," in 2020 23rd International Symposium on Wireless Personal Multimedia Communications (WPMC), 2020, pp. $1-6$.

[6] 3GPP, "Study on channel model for frequencies from 0.5 to $100 \mathrm{GHz}$ (Release 16)," 3rd Generation Partnership Project (3GPP), Technical Specification (TS) 38.901, 12 2019, version 16.1.0. [Online]. Available: https://portal.3gpp.org

[7] C. B. Sullivan and A. A. Kaszynski, "Pyvista: 3d plotting and mesh analysis through a streamlined interface for the visualization toolkit (vtk)," Journal of Open Source Software, vol. 4, no. 37, p. 1450, 2019. [Online]. Available: https://doi.org/10.21105/joss.01450

[8] Python Software Foundation, "Unit testing framework." [Online]. Available: https://docs.python.org/3/library/unittest.html

[9] Visicom. (2019) Geospatial Data. [Online]. Available: https://visicomdata.com/samples

[10] QGIS Development Team, QGIS Geographic Information System, QGIS Association, 2021. [Online]. Available: https://www.qgis.org

[11] GDAL/OGR contributors, GDAL/OGR Geospatial Data Abstraction software Library, Open Source Geospatial Foundation, 2021. [Online]. Available: https://gdal.org 